\title{
Screening for ACTH-dependent hypercortisolism in patients with pituitary incidentaloma
}

\section{David J Torpy}

Endocrine and Metabolic Unit, Royal Adelaide Hospital, University of Adelaide, Adelaide, South Australia 5000 Australia
Correspondence should be addressed to D J Torpy

Email david.torpy@health.sa.gov.au
Definitive diagnosis of mild hypercortisolism requiring treatment, without clinically evident Cushing's syndrome, described as 'pre-clinical Cushing's', represents a considerable challenge. The fact that the diagnosis of Cushing's syndrome is often delayed by several years $(1,2)$ is not surprising, given that the early features are characteristically non-specific. However, detection in this pre-clinical phase offers the theoretical potential to prevent Cushing's syndrome or reduce cortisol levels, which may otherwise act to accelerate cardiovascular disease (through blood pressure, lipids, and dysglycaemia) and ageing (through atrophic effects on skin, muscle and bone) and also have adverse neurocognitive effects.

Clinical Cushing's syndrome is rare with an incidence of $3-10 /$ million per year with the majority of cases arising from an adrenocorticotropin (ACTH)-secreting pituitary adenoma. In contrast, pituitary adenomas are very common with frequencies of $10 \%$ at autopsy and $10-38 \%$ by magnetic resonance imaging $(3,4)$.

Toini et al. (5) screened 68 patients with pituitary incidentalomas using the 1-mg overnight dexamethasone suppression test (DST) and a cortisol cut-off set at 50 nmol/1 - 7/68 were abnormal with post-dexamethasone cortisol levels 64-505 nmol/l. One patient had low ACTH and excess adrenal cortisol. Of the remaining six patients, five did not exhibit normal suppression to a $2 \mathrm{mg} 2$-day low-dose DST (LDDST) and were classified as having ACTH-dependent hypercortisolism, with an additional requirement for either an elevated 24-h urinary free cortisol (UFC), present in three of the four tested in the 1.1- to 2.7-fold normal range, or elevated midnight plasma ( $n=1$, twofold normal) or salivary $(n=3,1.1$ - to 1.7 -fold normal) cortisol in all the four tested.

The five patients (three males and two females) classified as having ACTH-dependent hypercortisolism had a mean age of 55 years ( $R \quad 37-82$ years) and comorbidities such as headaches $(n=2)$, ischaemic stroke $(n=1)$, mood disorders $(n=2)$ and hypertension considered essential in origin $(n=2)$. Two had macroadenomas $(13,44 \mathrm{~mm})$ with visual field defects and three had microadenomas. All had second-line testing (CRH or DDAVP stimulation, high-dose DST) and inferior petrosal sinus sampling in one patient, where it was necessary to exclude ectopic ACTH excess. Only three of the five patients with hypercortisolism underwent surgery as, of the remaining two patients, one refused to undergo surgery and another was unfit for surgery, and all had ACTH-positive immunohistochemistry of their adenomas. In addition, all three operated patients had low morning cortisol levels for 2-6 months while on replacement dose short-acting glucocorticoids, although one of these, who had stalk compression-related hyperprolactinaemia preoperatively, was rendered panhypopituitary by surgery.

Overall, biochemical testing was indicative of mild hypercortisolism in the five patients, universally so in the case of the required LDDST. Surgical findings of ACTH immunoreactivity in all three operated cases and isolated secondary hypocortisolism in two of the three patients are consistent with a pituitary tumour-driven cause for the mild hypercortisolism.

There are, however, important caveats regarding the testing, such as the limited specificity (85-95\%) of standard tests for hypercortisolism (1-mg overnight DST, 24-h UFC and late-night salivary cortisol (LNSC)) (6). The diagnostic accuracy of these tests is further challenged by their use in low pre-test probability cases who are older and have more comorbidities than the often used healthy controls in establishing test accuracy $(7,8,9)$. ACTHpositive immunochemistry is seen in up to one-third of clinically non-functioning pituitary adenomas, leading to

Published by Bioscientifica Ltd. 
the description of 'silent corticotropinomas' with reputed effects on tumour growth $(10,11)$. Hence, the presence of ACTH immunopositivity cannot be used to retrospectively validate the biochemical testing in individuals or small numbers of cases. Prolonged isolated hypocortisolism postoperatively is a guide to the presence of tumourdriven hypercortisolism and was evident in two of the three operated cases. However, hypocortisolism has been seen after resection of adrenal incidentalomas even after completely normal testing for hypercortisolism, suggesting that tumour-driven autonomous cortisol secretion rather than hormone excess may be sufficient to predispose to postoperative hypocortisolism (12).

Despite the limitations of available tools to diagnose mild Cushing's syndrome, it is likely that this study, a systematic protocol directed at mild hypercortisolism in pituitary incidentalomas, did uncover a rate of $4-7 \%$ of mild hypercortisolism in patients with pituitary incidentaloma.

Of course, we cannot conclude that this testing regimen detected Cushing's syndrome, as experienced clinicians did not find evidence of the syndrome on clinical assessment, for example, four of the five patients had a normal BMI. Crucially, among the patients with mild hypercortisolism, these clinicians did not detect evidence of Cushing's syndrome progression over a mean follow-up period of 30 months (51). Hypercortisolism with few or no clinical features of Cushing's syndrome or 'subclinical Cushing's' is a state for which there is no precise biochemical definition.

Current recommendations for screening for cortisol excess in pituitary incidentalomas vary, with the 2011 Endocrine Society Guidelines suggesting screening for hypercortisolism in patients where Cushing's syndrome is clinically suspected (13) and other guidelines suggesting that testing for cortisol excess using 24-h UFC should be restricted to those with macroadenomas when there is no clinical evidence of Cushing's syndrome (3). These recommendations have been made in the absence of large systematic studies of screening for hypercortisolism in pituitary incidentaloma.

Screening for Cushing's syndrome among patients with obesity has highlighted the difficulties with falsepositive test results (14). Initial enthusiasm for screening for Cushing's syndrome among patients with type 2 diabetes following reports of rates as high as $9.4 \%$ have been tempered by subsequent negative studies (15). At present, screening for Cushing's syndrome among patients with metabolic abnormalities representative of a single feature of Cushing's syndrome is not justified.
The clinical diagnosis of Cushing's syndrome should be based on the presence of a number of features appearing together in time, with evidence of progression over a period of follow-up, followed by testing for hypercortisolism. Manifestations of Cushing's syndrome include weight gain with central adiposity, neuropsychological disturbance, metabolic syndrome-type features such as hypertension, hyperglycaemia and dyslipidaemia, and the eventual development of atrophic features such as skin thinning with bruising and risk of infections, muscle wasting and osteoporosis with vertebral and distal lower limb fractures. As equivocal results are common in testing for hypercortisolism, near-normal cortisol values need to be considered carefully in the clinical context and repeated and related to other tests for hypercortisolism to avoid over-diagnosis (4).

This study of pituitary incidentalomas recalls the experience with adrenal incidentalomas, where abnormalities of the hypothalamic-pituitary-adrenal (HPA) axis are frequent, such as non-suppression of cortisol to dexamethasone, elevated UFC, low ACTH and low DHEAS levels, and where various combinations of abnormalities have been used to diagnose subclinical Cushing's syndrome, with frequencies of up to $9 \%$ of cases (16). The ongoing difficulty is determining the level of cortisol elevation that is clinically significant and would justify surgery, despite the association between elevated cortisol levels and metabolic abnormalities such as hypertension, dyslipidaemia and abdominal obesity, and some data showing improvements in these cardiovascular risk factors postoperatively (17).

Hypercortisolism, measured in the form of integrated cortisol measures (urine or saliva), disturbed cortisol circadian rhythm or resistance to dexamethasone suppression can follow a range of physiological perturbations, including stressors such as infection, trauma or psychic threat, starvation such as that seen in anorexia nervosa or pregnancy due to placental CRH secretion. In addition, hypercortisolism may be observed in pathological conditions unrelated to tumours affecting HPA axis function, such as melancholic depression, alcohol excess, together representing forms of 'pseudocushing's syndrome', following prenatal glucocorticoid excess and in ageing and dementia, perhaps related to atrophy of the hippocampi, which have a negative regulatory influence on the CRH neuron (18). Chronic stress may lead to adaptive processes that elevate glucocorticoids in the adrenal cortex and facilitate catecholamines form the sympathetic nervous system to produce an altered homoeostasis or allostasis, which may be adaptive, but have negative longterm consequences on metabolic and neurobehavioural systems known as allostatic load (19). Tests to exclude 
pseudocushing's syndrome have been proposed, such as the dexamethasone/CRH and desmopressin tests, but they have not been evaluated in all conditions associated with hypercortisolism and our capacity to rule out that pseudocushing's syndrome is limited $(20,21)$.

Clinically, unexpected isolated hypocortisolism may occur in patients with pituitary incidentalomas being excised for structural reasons, presenting with hypotension and nausea. This may be due to unanticipated ACTH secretion, which may not elevate but only replace and suppress normal corticotroph function.

A fundamental question is whether we should recommend routine detailed HPA axis testing in patients with pituitary incidentaloma and no evidence of clinical Cushing's syndrome?

Such an approach unequivocally has risks, namely potential over-diagnosis of hypercortisolism due to the known high false-positive rates of the principal Cushing's syndrome screening tests (1-mg overnight DST, 24-h UFC and LNSC), requiring repeated testings using different methodologies. Further, the detection or suspicion of hypercortisolism may lead to surgery that may be potentially unnecessary and expose patients to unnecessary surgical risks, such as hypopituitarism in the longer term or one of the more immediate risks of surgery such as ' $\mathrm{CSF}^{\prime}$ leak, haemorrhage and infection. Finally, the morbidity and mortality benefits of detection of subtle hypercortisolism from pituitary incidentaloma are unknown.

Hence, the study by Toini et al. (5) represents an important contribution to knowledge, but further studies to address the risks and benefits of detailed HPA axis testing for detection of hypercortisolism in pituitary incidentalomas are required before any change to current recommendations for the investigation of pituitary incidentalomas can be justified.

Given the travails of biochemical testing in hypercortisolism, we should perhaps recall the quote:

'We need to learn to measure what we value, not value what we can easily measure'

Marcus Aurelius (AD121-180)

\section{References}

1 Bolland MJ, Holdaway IM, Berkeley JE, Lim S, Dransfield WJ, Conaglen JV, Croxson MS, Gamble GD, Hunt PJ \& Toomath RJ. Mortality and morbidity in Cushing's syndrome in New Zealand.
Clinical Endocrinology 201175 436-442. (doi:10.1111/j.1365-2265. 2011.04124.x)

2 Valassi E, Santos A, Yaneva N, Tóth M, Strasburger CJ, Chanson P, Wass JA, Chabre O, Pfeifer M \& Feelders RA. The European Registry on Cushing's syndrome: 2-year experience. Baseline demographic and clinical characteristics. European Journal of Endocrinology 2011165 383-392. (doi:10.1530/EJE-11-0272)

3 Snyder PJ. Incidentally discovered sellar masses (pituitary incidentalomas). UpToDate 2013. Accessed January 2015.

4 Nieman LK, Biller BM, Findling JW, Newell-Price J, Savage MO, Stewart PM \& Montori VM. The diagnosis of Cushing's syndrome: an Endocrine Society Clinical Practice Guideline. Journal of Clinical Endocrinology and Metabolism 200893 1526-1540. (doi:10.1210/jc. 2008-0125)

5 Toini A, Dolci A, Verrua E, Malchiodi E, Sala E, Lania A, Chiodini I, Beck-Pecoz P, Arosio M, Spada A et al. Screening for ACTH-dependent hypercortisolism in patients affected with pituitary incidentaloma. European Journal of Endocrinology 2015172 363-369. (doi:10.1530/ EJE-14-0599)

6 Percori-Giraldi F, Ambroglio AG, De Martin M, Fatti LM, Scacchi M \& Cavagnini F. Specificity of first line tests for the diagnosis of Cushing's syndrome; assessment in a large series. Journal of Clinical Endocrinology and Metabolism 200792 4123-4129. (doi:10.1210/jc.2007-0596)

7 Liu H, Bravata DM, Cabacam J, Faff H \& Ryzen E. Elevated late-night salivary cortisol levels in elderly male type 2 diabetic veterans. Clinical Endocrinology 200563 642-649. (doi:10.1111/j.1365-2265. 2005.02395.x)

8 Montwill J, Igoe D \& McKenna TJ. The overnight dexamethasone suppression test is the procedure of choice in screening for Cushing's syndrome. Steroids 199459 296-298. (doi:10.1016/0039-128X (94)90116-3)

9 Isodori AM, Kaltsas GA, Mohammed S, Morris DG, Jenkins P, Chew SL, Monson JP, Besser GM \& Grossman AB. Discriminatory value of the low-dose dexamethasone suppression test in establishing the diagnosis and differential diagnosis of Cushing's syndrome. Journal of Clinical Endocrinology and Metabolism 200388 5299-5306. (doi:10.1210/jc. 2003-030510)

10 Pawlikowski M, Kunert-Radek J \& Radek M. Silent corticotropinoma. Neuro Endocrinology Letters 200829 347-350.

11 Bradley KJ, Wass JA \& Turner HE. Non-functioning pituitary adenomas with psotive immunostaining for ACTH behave more aggressively than ACTH immunonegative tumours but do not recur more frequently. Clinical Endocrinology 200358 59-64. (doi:10.1046/j.1365-2265.2003. 01674.x)

12 Eller-Vainicher C, Morelli V, Salcuni AS, Torlontano M, Coletti F, Iorio L, Cuttitta A, Ambrosio A, Vicentini L \& Carnevale V. Postoperative hypocortisolism after removal of an adrenal incidentaloma: is it predictable by an accurate endocrinological work-up before surgery? European Journal of Endocrinology 2010162 91-99. (doi:10.1530/EJE-09-0775)

13 Freda PU, Beckers AM, Katznelson L, Molitch ME, Mantori VM, Post KD \& Vance ML. Pituitary incidentaloma: an Endocrine Society Clinical Practice Guideline. Journal of Clinical Endocrinology and Metabolism 2011 96 894-904. (doi:10.1210/jc.2010-1048)

14 Baid SK, Rubino D, Sinali N, Ramsey S, Frank A \& Nieman LK. Specificity of screening tests for Cushing's syndrome in an overweight and obese population. Journal of Clinical Endocrinology and Metabolism 200994 3857-3864. (doi:10.1210/jc.2008-2766)

15 Tabarin A \& Perez P. Pros and cons of screening for occult Cushing's syndrome. Nature Reviews. Endocrinology 20117 445-455. (doi:10.1038/ nrendo.2011.51)

16 Mantero F, Terzolo M, Arnaldi G, Osella G, Masini AM, Giovagnetti M, Opocher G \& Angeli A. A survey on adrenal incidentaloma in Italy. Study group on adrenal tumors of the Italian Society of Endocrinology. Journal of Clinical Endocrinology and Metabolism 2000 85 637-644. 
17 Toniato A, Merante-Boschin I, Opocher G, Pelizzo MR, Schiavi F \& Ballotta E. Surgical versus conservative management for subclinical Cushing syndrome in adrenal incidentalomas: a prospective randomized study. Annals of Surgery 2009249 388-391. (doi:10.1097/ SLA.0b013e31819a47d2)

18 Chrousos GP. Stress and disorders of the stress system. Nature Reviews. Endocrinology 20097 374-381. (doi:10.1038/nrendo.2009.106)

19 Karatsoreos IN \& McEwen BS. Psychobiological allostasis: resistance, resilience and vulnerability. Trends in Cognitive Sciences 201115 576-584. (doi:10.1016/j.tics.2011.10.005)
20 Yanovski JA, Cutler GB Jr, Chrousos GP \& Nieman LK. Corticotropinreleasing hormone stimulation following low-dose dexamethasone administration. A new test to distinguish Cushing's syndrome from pseudocushing's states. Journal of the American Medical Association 1993 269 2232-2238. (doi:10.1001/jama.1993.03500170062035)

21 Percori Giraldi F, Pivonello R, Ambrogio AG, De Martino MC, De Martin M, Scacchi M, Toja PM, Lombardi G \& Cavagnini F. The dexamethasone-suppressed corticotropin-releasing hormone test and the desmopressin test to distinguish Cushing's syndrome from pseudocushing's states. Clinical Endocrinology 200766 251-257.

Received 16 January 2015

Accepted 20 January 2015 